\title{
Consideration of analogies between magnetic and quantum notices for molecular network
}

\author{
Henryk Piech ${ }^{1}$, Jerzy Jelonkiewicz ${ }^{2 *}$, Lukasz Laskowski ${ }^{3}$, Magdalena Laskowska ${ }^{4}$ \\ ${ }^{123}$ Czestochowa University of Technology, PL-42-201 Czestochowa, Poland \\ ${ }^{4}$ Institute of Nuclear Physics Polish Academy of Sciences, PL-31342 Krakow, Poland
}

\begin{abstract}
Magnetic properties of spin glass materials $[9,13]$ are close to quantum interpretation in their nature description [17]. Therefore, we can look for possible kinds of analogies in process of defining theoretic and practice conventions, rules and applications of the specific characteristics in elaboration quantum calculation strategies. We have not investigated possibilities to create directly quantum calculation units and practice calculation structures like qubits, registers, gates etc. [4,18], but dealing with spin and quantum definitions and descriptions we can try to involve these notices from different domains. Such a pragmatic approach only intuitively gives chances to create the transition theory and implement it even partially. Obviously, almost all of us have heard about quantum factorization, cryptography or teleportation but it is obtained as a result of exploration casually selected quantum properties and adapting them to mathematic problems. In our approach, we carefully investigate involutions among spin and quantum nature looking at possible implementation in molecular network.
\end{abstract}

\section{Introduction}

One of essential problems connected with magnetic spin quantification consists in finding clear and readable approach leading to adequate physical and quantum mechanical characteristics and functions description $[5,14,16]$. Starting with selection of relevant structures and parameters according to quantum conversion, the mutual involvement can be exposed and practically exploited [3,9]. Widely explored experiments give us more and more information about magnetic properties of different kinds of material (spin-glass, paramagnetic, ferromagnetic etc.) molecules $[2,8,13,17]$. The result of experiments based on, e.g., Raman and EDX spectra $[6,10,11]$ are agreed with theoretical analysis what confirm correctness of the models for supplementary investigations also theoretic. Carried out research can lead, for example, to conclusions about homogenous distribution of active units inside silica matrix [7,10]. On the one hand, the probabilistic aspect has to be regarded in quantum and spin magnetic processes $[5,6]$ but on the other hand the parallel activity nature or maybe, in future, conversion is inherent feature of these conventions. In both cases (spin magnetic and quantum conventions) we should avoid quantization problem [15]. Presentation and explanation of new ideas can be graphically illustrated as selected superposition of chronology and space. We should talk about quantum calculi considering gates, Boolean function operations or computation as one with enough information on the output to deduce unambiguously from the input values [12]. Such operations on gates are essential for quantum computing also because of the quantum physics phenomena reversibility $[16,20,21]$. For theoretical physics quantum computing can be seen as a new challenge and also a new source of stimuli aims and paradigms how to deal with one of the most basic problems for contemporary science (physics) [17,19]. The big question is: how to resolve mysteries paradoxes and quite evident incompleteness of the most basic powerful and fascinating theory in physics, i.e. quantum theory and perhaps how to develop the main global aim of the current science, a new global theory of everything. For experimental physics especially in the area of atomic optic physics and mechanics quantum computing brings radically new challenges. We have omitted the basic ideas of classical and quantum computing including description of some experiments principles of quantum mechanics and basics of Hilbert spaces. We will start with a basic question: why to consider quantum computing? This involves introduction of basic ideas of quantum computing via a comparison of quantum and randomized computing [4,21]. Finite dimensional Hilbert spaces correspond to the example of such particle properties as spin value, polarization, position or momentum. Our proposition refers to a suggestion that outside magnetic field can play role of a controller of character logic operations, implemented by magnetic spin gates.

\footnotetext{
* Corresponding author: jerzy.jelonkiewicz@iiisi.pcz.pl
} 


\section{Base formalisms representing quantum calculation in relation to spin properties presentation}

Qubit is a quantum state of two level own orientations $\mid 0>$ and $\mid 1>$, which can be presented as superposition of inner states:

$$
\begin{aligned}
& |\mathrm{Y}>=\mathrm{a}| 0>+\mathrm{b} \mid 1> \\
& |\mathrm{a}|^{2}+|\mathrm{b}|^{2}=1
\end{aligned}
$$

Qubit can be illustrated like spin in Bloch sphere (Fig.1 and 2)[4].
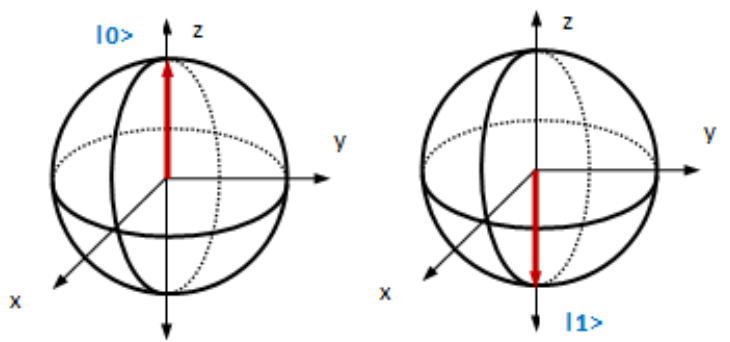

Fig. 1. Qubit illustrations: $|a|^{2}=1$ and $|\mathrm{b}|^{2}=0$ (left) as well as $|a|^{2}=0$ and $|\mathrm{b}|^{2}=-1$ (right)

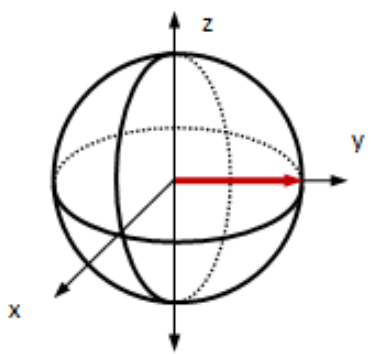

$\frac{1}{\sqrt{2}}(|0\rangle+|1\rangle)$

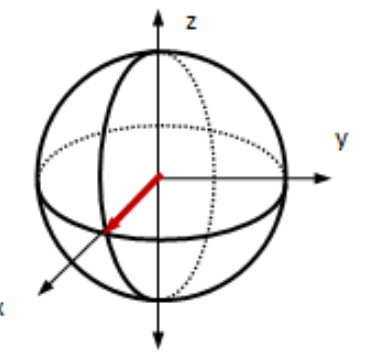

$\frac{1}{\sqrt{2}}(|0\rangle+i|1\rangle)$
Fig. 2. Qubit illustrations: $|\mathrm{a}|^{2}=1 / 2$ and $|\mathrm{b}|^{2}=1 / 2$ (left) as well as $|a|^{2}=1 / 2$ and $|b|^{2}=-1 / 2$ (right).

Physically we can treat qubit as a spin in magnetic field being superposition of the base states. Two level quantum state (Fig.2) has the form:

$$
|\psi\rangle=\cos \left(\frac{\theta}{2}\right)|0\rangle+e^{i \phi} \sin \left(\frac{\theta}{2}\right)|1\rangle,
$$

where $e^{i f}$ - phase coefficient, $\mathrm{f}, \mathrm{q}$ - real value of phases.

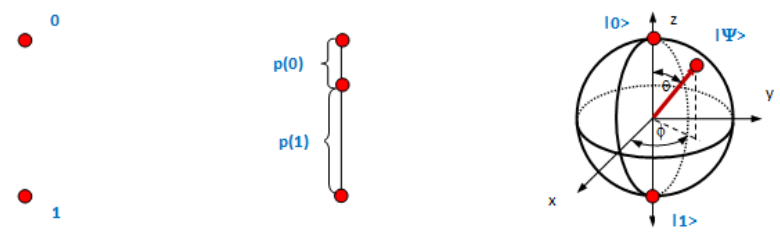

Fig.2. The classic, probabilistic (spin) and quantum states

The short explanation of spin behavior can be presented as follows: we start with chaotic spin vectors. Rotating around their own axe protons create magnetic spin moment. Total vector of magnetic spins is equal zero. Then we switch on an outside magnetic field B (Fig.3).
The part of spin vectors will allocate along field B while remained in opposite direction.

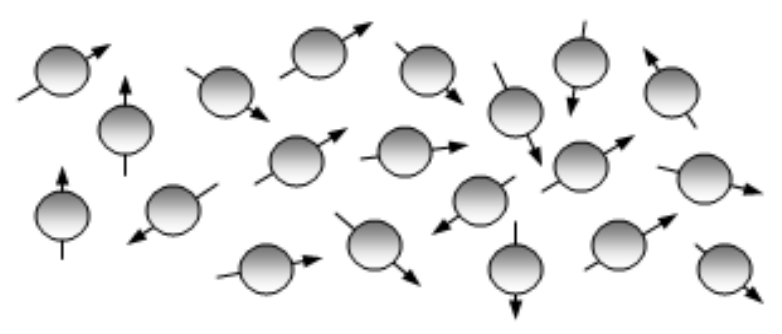

Chaotic stage

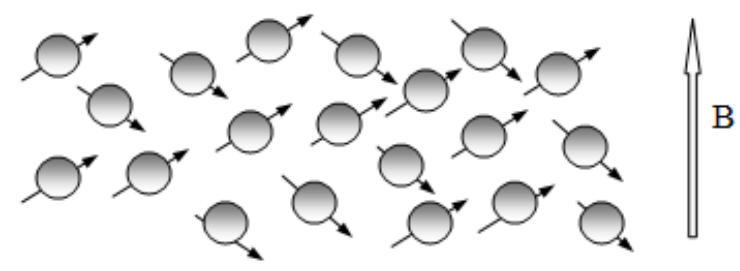

Magnetic field interference

Fig. 3. The illustration of magnetic field influence on spin vector orientation

All spinning protons are in precession, cyclic movement (Fig. 4). The speed of this motion is usually described by frequency (called Lamor frequency) which is equal to the frequency of absorbed by nucleus radiation.

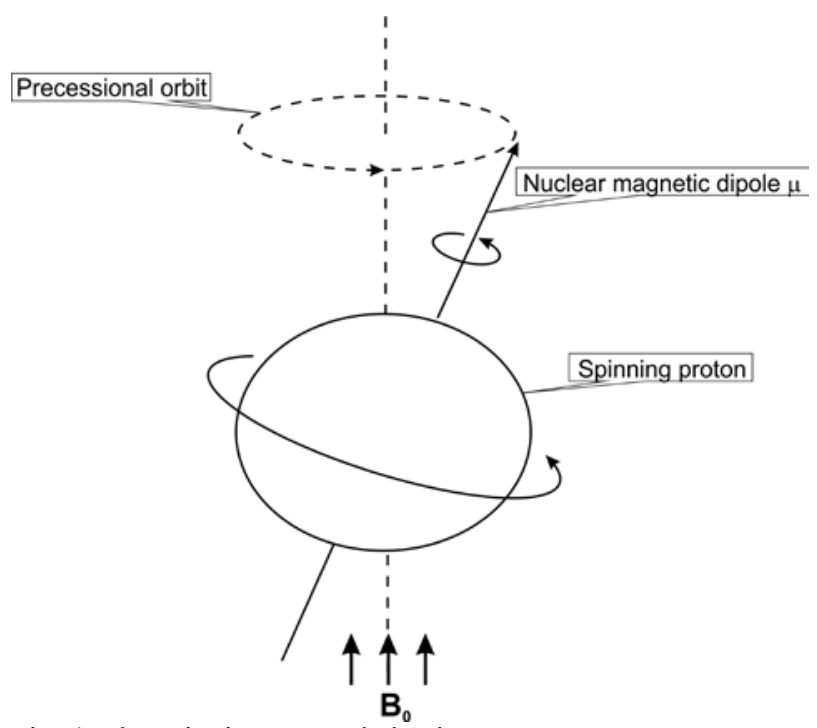

Fig. 4. The spinning proton behavior

When the field $\mathrm{B}_{1}$ is on the magnetic vector rotates around axe x (Fig. 5a). Its new location presents Fig. 5b. After turn off radiation magnetic vector returns to its previous location (Fig. $5 \mathrm{c}$ ). 


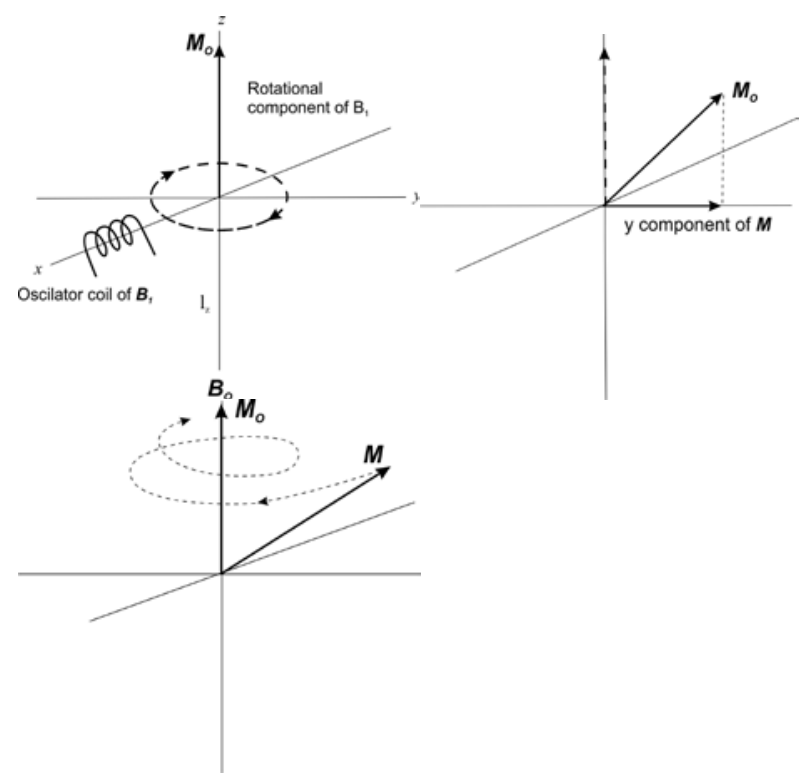

Fig 5. Magnetic vector rotations before(a), during (b) and after radiation $(\mathrm{c})$.

Its probability calculation we can write: $\left.\mathrm{p}(1, \mathrm{k})=\left(\mathrm{M}_{0}+\mathrm{M}_{0} \cos (\theta+\Delta \theta)\right) \quad /\left(2 \mathrm{M}_{0}\right)\right)$, where $\mathrm{p}(1, \mathrm{k}) \quad-$ probability of state " 1 " referred to k-th molecule, $\theta-$ phase correction inferring from magnetic field impact (Fig.6). Obviously $\mathrm{p}(0, \mathrm{k})=1-\mathrm{p}(1, \mathrm{k})$.

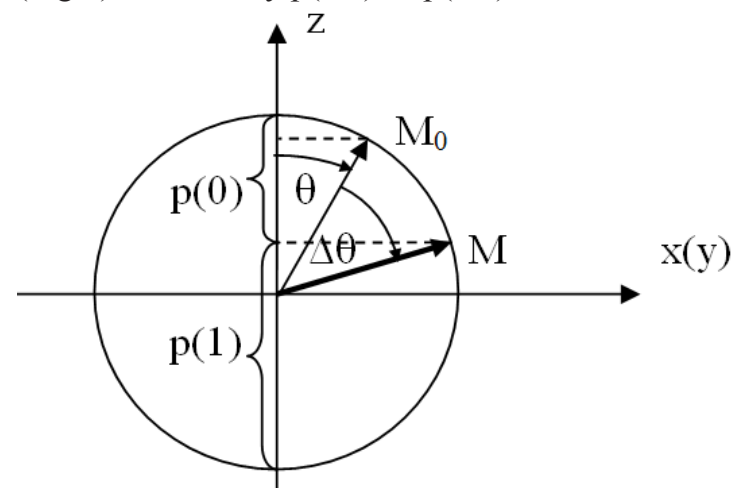

Fig. 6. Variant A of evaluation state probability

In another variant (B) we use formula: $\mathrm{p}(1, \mathrm{k})=\left(\mathrm{M}_{0} \cos (\theta)+\mathrm{M}_{0} \cos (\Delta \theta)\right) /\left(2 \mathrm{M}_{0} \cos (\theta)\right)$ (Fig. 7).

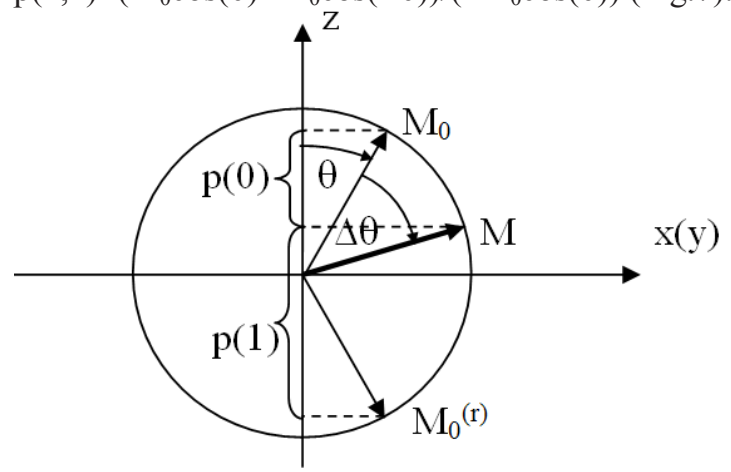

Fig. 7 Variant B of evaluation state probability

Including relatively magnetic spin changing (according to neutral location) we can propose variant $\mathrm{C}$, which is represented by the formulas: $\mathrm{p}(1, \mathrm{k})=\left(\mathrm{M}_{0}-\mathrm{M}_{0} \cos (\theta+\Delta \theta)\right)$ $/ \mathrm{M}_{0},-\theta \leq \Delta \theta \leq 90-\theta$, or $\mathrm{p}(1, \mathrm{k})=\left(\mathrm{M}_{0}+\mathrm{M}_{0} \cos (\theta+\Delta \theta)\right) / \mathrm{M}_{0}$, $90-\theta \leq \Delta \theta \leq 180-\theta$ (Fig. 8)
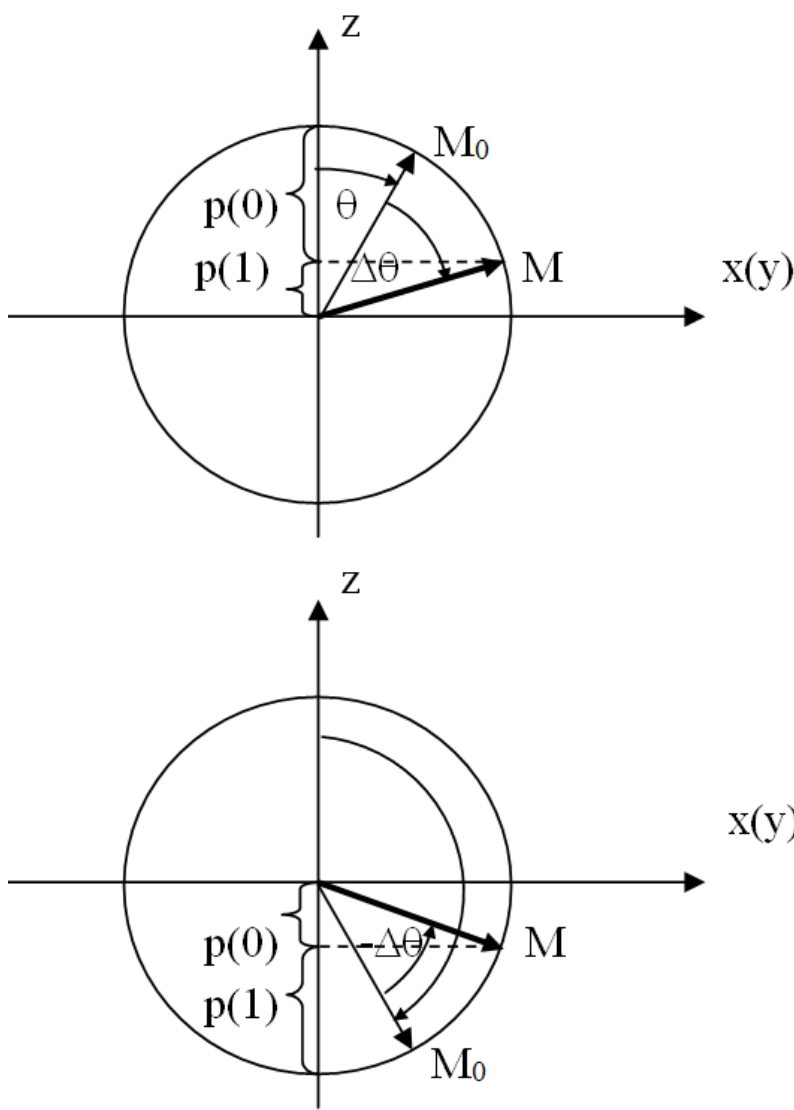

Fig. 8 Variant $\mathrm{C}$ of evaluation state probability

Obviously many different forms can be used for defined dependence considering probabilities spin information and magnetic state values. This information is the base of transition to quantum conversion description.

\section{Generic strategy of energy spin modeling}

Usually we start with Edwards-Anderson model [1]. For generalization Hamiltonian sum for all spin $S$ combination is defined as:

$$
H(S, J)=-\sum_{\|i-j\|=1} J(i, j) * S(i) * S(j)
$$

where $\|*\|$ - Euclidian distance, $\boldsymbol{J}=\{\boldsymbol{J}(\boldsymbol{i}, \boldsymbol{j})\}$ - matrix of spin influences, $S(i)=\{+1,-1\}$

There are much more information available for this model but they are not important for our consideration. We supplement information about next models, for example using Sherrington-Kirkpatric model:

$$
H(S, J)=-\sum_{(i, j)} J(i, j) * S(i) * S(j)-h \sum_{i} S(i)
$$

where $S(i)=1, \ldots, N, \quad \boldsymbol{p}(\boldsymbol{J}(\boldsymbol{i}, \boldsymbol{j})) \cong \boldsymbol{e}^{-\boldsymbol{N J}^{\mathbf{2} / 2 \boldsymbol{J}^{2}}} \quad h \quad$ influences of outside field energy on single spin. 
The same formula is used in Ising model but $(i, j)$ refers to pair of adjacent spins $[2,7,8,19]$. The average values of $\overline{\boldsymbol{h}(\boldsymbol{i})}=\mathbf{0}$ as well as $\overline{\boldsymbol{h}(\boldsymbol{i}) \boldsymbol{h}(\boldsymbol{j})}=\Delta \boldsymbol{S}(\boldsymbol{i}, \boldsymbol{j})$.

Magnetization degree is equal to $\mathrm{M}=\frac{1}{N} \sum_{\mathbf{1}}^{N} S(i)$

In $\mathrm{p}$-spin model the following convention is used:

$H(S, J)=$

$$
-\sum_{i(1), i(2), \ldots, i(r)} J(i(1), \ldots, i(p)) * S(i(1)) * S(i(2) * \ldots * S(i(r))
$$

$$
\sum_{\text {where }} S(i)=N \quad, p(J) \cong e^{-\frac{J^{2} N^{r-1}}{r !}}
$$

For the above equations all spin influences are regarded. We can also consider independent pair model. To describe independent connection usually correlation function is used:

$f(i, j)=<S(i) * S(j)>-<S(i)>*<S(j)>$,

where $<S(i)>$ means average over $i$-th spin states. Basing on statistic sum formalisms we get:

$$
<S(0) * S(1)>=\sum_{\sigma} \frac{1}{Z} e^{-\beta^{*} E_{01}(\sigma)} S(0) * S(1) * S(1) * S(2),
$$

where $\mathrm{Z}=\sum_{\sigma} \boldsymbol{e}^{-\beta * E_{\sigma}}$ - statistical sum, $\sigma_{\text {- state, }} \boldsymbol{E}_{\sigma}$

$$
\text { - state } \sigma \text { energy, } \beta=\frac{1}{\boldsymbol{k T}}, k \text { - the number or adjacent }
$$
molecules, $T$ - temperature.

In the random energy model there are $2^{\mathrm{N}}$ configurations whose energies $\left\{E_{i}\right\}$ are independent random variables and laid out accordingly with distribution:

$$
p\left(E_{i}\right)=\mathrm{C} * e^{-\frac{E_{i}^{2}}{N}},
$$

where $i=1,2, \ldots, 2^{N}, \mathrm{C}$ - normalization factor

All models refer to net structures and inner connection between them. These nets can be casual, pseudo-regular or regular $[8,19]$. For these type of nets main energetic and additional topologic parameters are defined. In our case parameters are not relevant but they have very important practical meaning.

\section{Quantum representation evolution}

States $\mid 0>$ and $\mid 1>$ of a qubit are referred to classical states (bits). The difference between classical bits and qubits is that: classical bit can be set up only to one of the two states while a qubit can take any quantum linear superposition of $\mid 0>$ and $\mid 1>$ in uncountable states space. This means that infinite amount of information could be encoded in amplitudes of a single qubit by appropriately choosing $\alpha$ and $\beta$. Going to two qubits, we present this situation as a superposition of four states:

$\left|\Psi>=\alpha_{00}\right| 00>+\alpha_{01}\left|01>+\alpha_{10}\right| 10>+\alpha_{11} \mid 11>$, where $\left|\alpha_{00}\right|^{2}+\left|\alpha_{01}\right|^{2}+\left|\alpha_{10}\right|^{2}+\left|\alpha_{11}\right|^{2}=1$.

During the measure process of two qubits state the probability that the $1^{\text {st }}$ qubit is in state $i$ and $2^{\text {nd }}$ in state $j$ is equal to $p(i, j)=\left|\alpha_{i, j}\right|^{2}$. The probability that the $1^{\text {st }}$ qubit is equal to 0 , independently of $2^{\text {nd }}$ qubit state equals $\mathrm{p}\left(1^{\mathrm{st}}\right.$ qubit $\left.=0\right)=\left|\alpha_{00}\right|^{2}+\left|\alpha_{01}\right|^{2}$. The new state consists of the terms in the superposition and in the normalized form can be represented by unit vector:

$$
\mid \phi>=\frac{\alpha_{00}\left|00>+\alpha_{01}\right| 01>}{\sqrt{\left|\alpha_{00}\right|^{2}+\left|\alpha_{01}\right|^{2}}}
$$

The joint state of the two qubits is described in the following way [21]:

$$
\begin{aligned}
& |\phi>=| \phi>\otimes \mid \phi>=\left(\alpha_{1}\left|0>+\beta_{1}\right| 1>\right) \otimes\left(\alpha_{2}\left|0>+\beta_{2}\right| 1>\right)= \\
& =\alpha_{1} \alpha_{2}\left|00>+\alpha_{1} \beta_{2}\right| 00>+\beta_{1} \alpha_{2}\left|00>+\beta_{1} \beta_{2}\right| 00>.
\end{aligned}
$$

The evolution of a quantum system has unitary character [4]. If we have a $s$-state quantum system then a unitary transformation is linear which can be represented by $s^{\prime} s$ matrix $U$. The rows and columns form orthogonal basis. $\mathrm{U}$ preserve inner product $\langle u \mid v\rangle$ between states $|u\rangle$ and $|v\rangle$, therefore $U$ preserves norms and angles. The eigenvalues of $U$ are all of the form $e^{i \theta}[4,21]$. $U$ has the diagonalized form:

The evolution of a quantum system has unitary character [4]. If we have a $s$-state quantum system then a unitary transformation is linear which can be represented by $s^{\prime} s$ matrix $U$. The rows and columns form orthogonal basis. $\mathrm{U}$ preserve inner product $\langle u \mid v\rangle$ between states $|u\rangle$ and $|v\rangle$, therefore $U$ preserves norms and angles. The eigenvalues of $U$ are all of the form $e^{i \theta}[4,21]$. $U$ has the diagonalized form:

$\left(\begin{array}{cccc}e^{i \theta_{1}} & 0 & \cdots & 0 \\ 0 & \cdots & \cdots & 0 \\ \cdots & \cdots & \cdots & 0 \\ 0 & \cdots & 0 & e^{i \theta_{s}}\end{array}\right)$

Let us go to unitary transforms (quantum gates).

The example of gate with one qubit is Hadamar gate (a reflection around $\pi / 8$ or rotation around $\pi / 4$ followed by a reflection [21]) .

$$
H=\frac{1}{\sqrt{2}}\left(\begin{array}{cc}
1 & 1 \\
1 & -1
\end{array}\right)
$$

$H$-is real and symmetric, $H^{2}=I$

More flexible to spin performing may occur rotation gate (according to $\theta$ ):

$$
U=\left(\begin{array}{cc}
\cos (\theta) & -\sin (\theta) \\
\sin (\theta) & \cos (\theta)
\end{array}\right)
$$

Next important gates are NOT gates:

$$
N O T=\left(\begin{array}{ll}
0 & 1 \\
1 & 0
\end{array}\right)
$$

and Phase Flip gates

$$
P F=\left(\begin{array}{cc}
1 & 0 \\
0 & -1
\end{array}\right)
$$


The basis contains states:

$$
\left|+>=\frac{1}{\sqrt{2}}(|0>+|->), \quad\right|->=\frac{1}{\sqrt{2}}(|0>-|->)
$$

and we obtain: $z|+>=|->$ and $z|->=|+>$.

Moreover, we can also mention about Controlled NOT (CNOT) gate:

$$
\text { CNOT }=\left(\begin{array}{llll}
1 & 0 & 0 & 0 \\
0 & 1 & 0 & 0 \\
0 & 0 & 0 & 1 \\
0 & 0 & 1 & 0
\end{array}\right)
$$

The first bit - control bit, the second - target bit. This gate changes qubit state if and only if the control bit is equal $\mid 1>$. Therefore, we obtain:

$$
C N O T|00>=| 00>, C N O T|01>=| 01>C N O T \mid 10>=
$$

$|11>, C N O T| 11>=\mid 10>$.

There are several variants of different controlled gate Toffoli, Fredkin, Petri etc. [4].

If we want to describe unitary transformation $k$-state system A into $l$-state system B we can use tensor product in a form $\mathrm{A}|\mathrm{i}>\otimes \mathrm{B}| \mathrm{j}>$, where $0 \leq i \leq k-1$ and $0 \leq j \leq l-1$ :

$$
\left.\left(\sum_{i=0}^{k-1} \alpha_{i} \mid x_{i}>\right)\left(\sum_{i=0}^{l-1} \beta_{j}\left|x_{j}\right\rangle\right)=\sum_{i=0}^{k-1 l-1} \sum_{j=0} \alpha_{i} \beta_{j}\left|x_{i}\right\rangle\left|y_{j}>=\sum_{i=0}^{k-1 l-1} \sum_{j=0} \alpha_{i j}\right| x_{i}\right\rangle\left|y_{j}\right\rangle
$$

So called untangled system and lexicographical matrix basis for two bits structures is the following:

$$
\left(\begin{array}{ccc}
a_{11} B & a_{12} B & a_{1 n} B \\
a_{21} B & a_{22} B & a_{2 n} B \\
a_{m 1} B & a_{m 2} B & a_{m n} B
\end{array}\right)
$$

where $\mathrm{B}=\left\{\mathrm{b}_{\mathrm{i}, \mathrm{j}}\right\}, 1 \leq i \leq k$ and $1 \leq j \leq l$.

\section{The effort of gates creation for spin nature elements}

In this section we do not describe synthesis process of microporos or mesoporous materials but only use them basing on their microstructures and features. The microstructure of mesoporous silica (SBA-15) in simplified form is presented in Fig. 9.

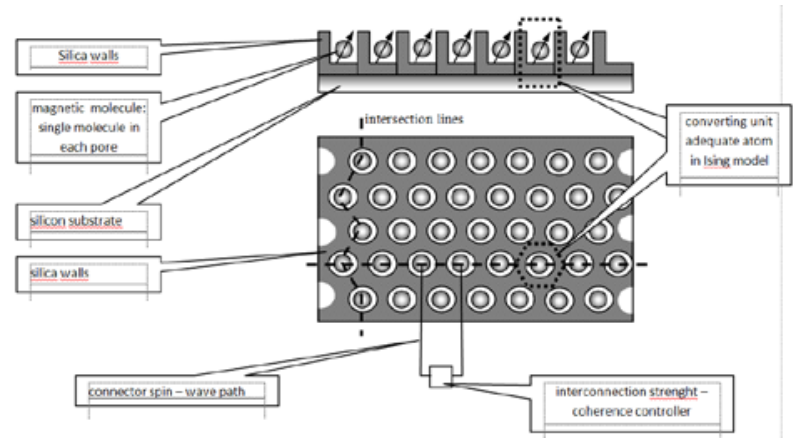

Fig.9. The structure model of mesoporous silica

Generally, magnetic molecules are mutually energetically isolated (SBA-15) or partially connected (ferromagnetic, paramagnetic materials), creating network with casual structure. Theoretically, it is possible to build energetic interconnection between molecules (coherent controller) based on spin-FET (field effect transistor design [1]) and create a molecular network. In spite of this, our proposition leads to similar solution. Basing on magnetic properties, we propose to apply influences outside energetic field on spin state and use these possibilities to encode probabilistic binary state. Consequently, we suggest the implementation of fundamental logic conversion performed by gates with a help of outside magnetic field controller. Let us consider the simplest logic operations (conjunction, disjunction). The idea of our proposition can be presented as follows: in initial state spin moment $M$ is not strictly defined, so we assume that its position (q) is as in Fig. 10.

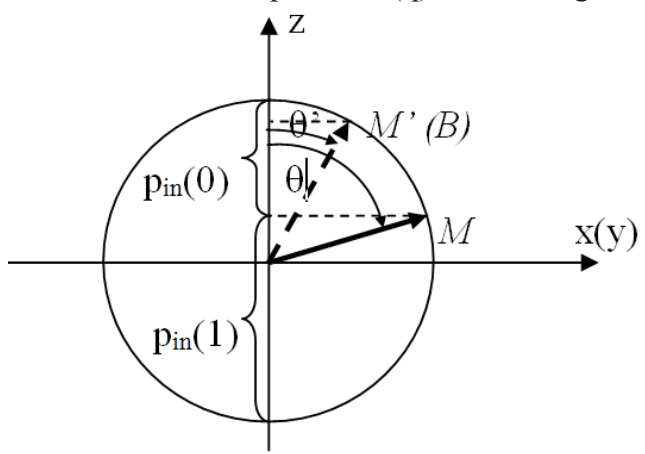

$\mathrm{B}^{\prime}$

Fig. 10 The effect of turning on outside magnetic field B' evoking phase correction $\theta^{\prime}<\theta$ and adequate probability correction $\mathrm{p}^{\prime}(1)>\mathrm{p}_{\text {in }}(1)$.

Turning on outside magnetic field B' we cause the change of value and phase of magnetic spin vector. After scaling procedure we will get the vector $M^{\prime}$ ' (dashed arrow in Fig.10). The vector $M$ ' refers to bit " 1 " (or to qubit $\mid 1>)$ with probability $\mathrm{p}^{\prime}(1)>\mathrm{p}_{\text {in }}(1)$. The angle $\theta$ depends on strength of magnetization. In the similar way we form information that refers to bit " 0 " (or adequate qubit) what is presented in Fig. 11. On this stage of consideration the value of spin vector do not play any relevant role.
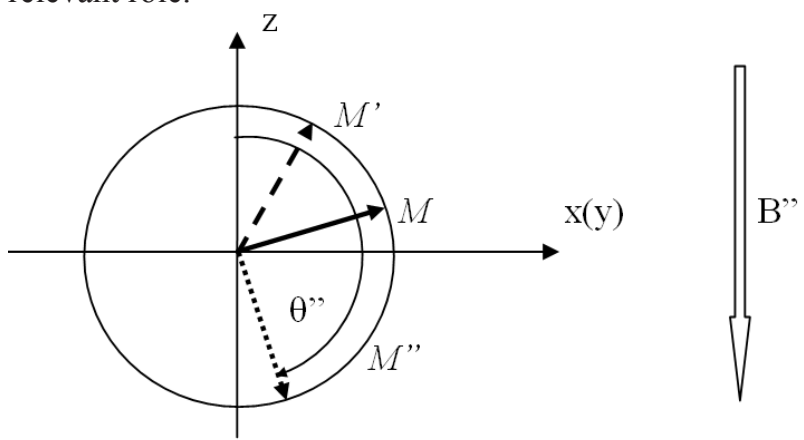

Fig. 11 The effect of turning on outside magnetic field B' evoking phase correction $\theta ">\theta$ and adequate probability correction p" $(0)>p_{\text {in }}(0)$.

Thus a spin vector $M$ " appears (doted arrow) that is adequate to " 0 " bit information. We assume, that absolute values of magnetization strength are in relation $\left|\mathrm{B}^{\prime \prime}\right|>\left|\mathrm{B}^{\prime}\right|$. Therefore, the phases are in relation $\left|\theta^{\prime}\right|>\mid 90$ $\theta$ '”. After turn off the field previous magnetic characteristics are preserved for relaxation time [13]. 
The next stage of gates building is connected with energetic interconnections in stochastic (or statistic) way. It is presented in a diagram as a general effect of mutual molecules influences to show the idea of gates creation. Firstly, this refers to conjunction gate functioning (Fig.12) in which main controller assumption is $\left|\theta^{\prime}\right|>\left|90-\theta^{\prime \prime}\right|$.

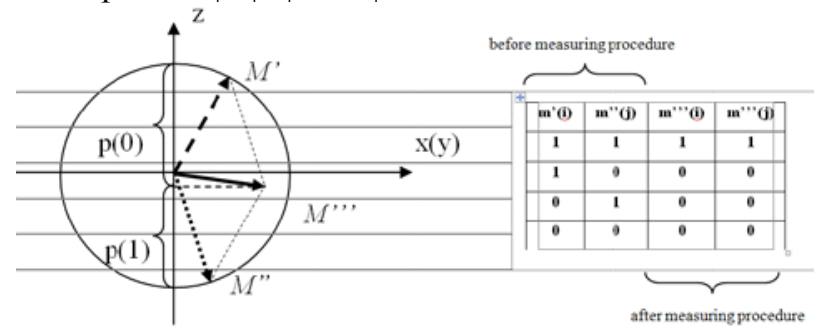

Fig.12 The conjunction effect (CC gate) on base of probability dependency: $(p(0)>p(1)) \sim$ bit " 0 " (qubit $\mid 0>), \quad(p(1)>p(0)) \sim$ bit "1" (qubit $\mid 1>$ ).

Secondly, we can refer to disjunction gates. In this case we assume that $\left|\theta^{\prime}\right|<\left|90-\theta^{\prime \prime}\right|$.

The adequate situation is presented in Fig.13.

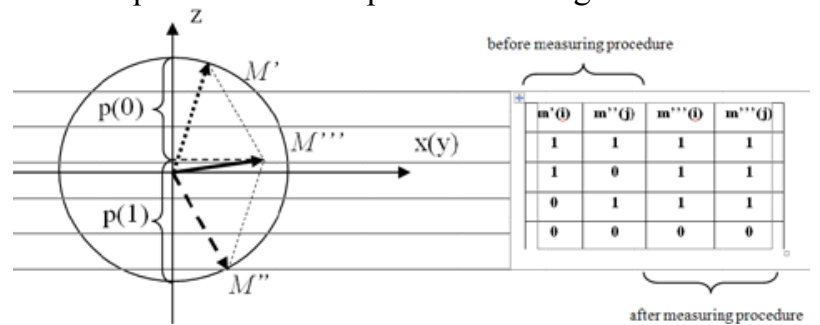

Fig.13 The conjunction effect (CD gate) on base of probability dependency: $(p(0)>p(1)) \sim$ bit " 0 " (qubit $\mid 0>),(p(1)>p(0)) \sim$ bit "1" (qubit $\mid 1>$ ).

To negate initial (or given) state we use the opposite directed energetic fields (Fig.14). The strength of its influences on spins should follow the condition $\theta$ "'>90$\theta$ '.

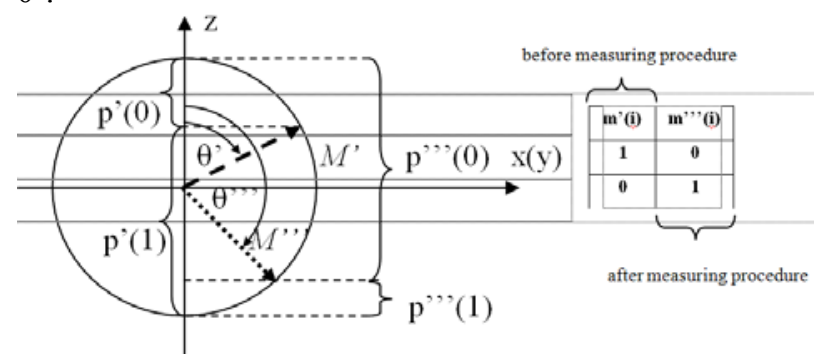

Fig.14 The negation gate $(\mathrm{CN})$ functioning with controlling by outside magnetic field

The graphic presentation of control spin gates can be similar as quantum gates (Fig. 15).

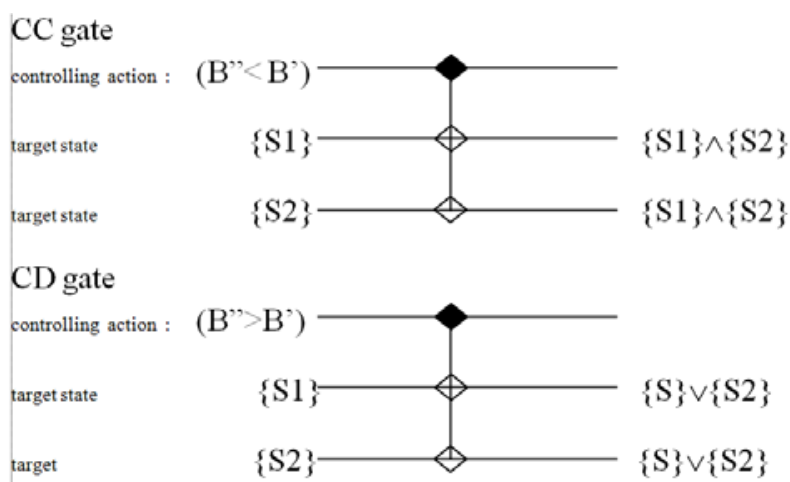

CN gate

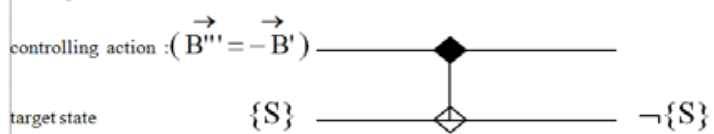

Fig. 15 The provisory of spin gate illustration.

Naturally, we assume that initial state of spin magnetic parameter is known or can be read or measured. In similar way we can project different kinds of gates.

\section{Conclusions}

We hope that finding analogies between quantum and spin nature of molecules exposed to magnetic field can shorten the distance between theory and practice. The proposition of using outside magnetic field to control character of logic conversion in stochastic approach offers a chance to quantum calculation possibilities. Technically this is much more difficult because of many implementation limits and constrains connected with spin nature character $[1,6]$. Selection of the materials, considered parameters and also sources of outside energy creates quite a large research space. Perhaps, proposed approach of creation controlled elementary logic gates guaranties combining the bests features of traditional, quantum and nanostructure convention leading to molecular network.

\section{References}

1. Agnihotri P., Bandyopadhaya S.: Analysis of the two dimensional data-das spin field effect transistor, Physica E: Low-dimensional Systems and Nanostructures 42(5),1736-1740 (2010)

2. Albayark E., Yigit A., and Akkaya S.: The antiferromagnetic Ising model for a bilayer Bethe lattice, Journal of Magnetism and Magnetic Materials 320 2241-2248, (2008)

3. Bonesteel N.E., Stepanenko D., and Di Vincenzo D.P.: Anisotropic spin exchange in pulsed quantum gates, qu-phys 0002092 (2000)

4. Brylinski L.L., and Brylinski R.: Universal quantum gates, qu-phys 0108062 (2001)

5. Childs A. and Chuang I.: Universal quantum computation with two-level trapped joins qu-phys 0008065 (2000)

6. Cornia A., Mannini M., Sainctavit P., Sessoli R.: Chemical strategies and characterization tools for 
the organization of single molecule magnets on surfaces, Chem. Soc. Rev. 40, 3076-3091 (2011)

7. Dorogovtsev S., Goltsev A.V., and Mendes J.F.F.: Ising model on networks with an arbitrary distribution of connections, Physical Review E 66:016104 (2002)

8. Herrero C.P.: Antiferromagnetic Ising model in small-world networks, Physical Review E 77:041102 (2008)

9. Lacroix C.: Magnetic properties of strongly frustrated and correlated systems, Physica B: Physic and Condensed Matter 404 3038-3041 (2009)

10. Laskowski L., Laskowska M.: Functionalization of SBA-15 mesoporous silica by cu- phosphonate units: Probing of synthesis route. Journal of Solid State Chemistry, 220, 221-226 (2014)

11. Laskowski L., Laskowska M., , Balanda M., Fitta M., Kwiatkowska J., Dzilinski K., Kaczmarska A: Mesoporouse silica SBA-15 functionalized by Nikel-phosphonic units: Raman and magnetic analysis. Microporous and Mesoporous Materials 200, 253-259 (2014)

12. Malarz K., Antosiewicz W., Karpińska J., Kułakowski K.: Avalanches in complex spin networks, Physica A 373, 785-795 (2007)

13. Parisi G.: Some consideration of finite dimensional spin glasses, Journal of Physics A 41:324002 (2008)

14. Piech H., Siedlecka-Lamch O.: Parallel Quantum Algorithm for finding the consistency of Saaty's matrices, Lecture Notes in Computer Science $\mathbf{7 2 0 3}$ 102-111 (2012)

15. Schlieman J., and Loss D.: Entanglement and quantum gate operations with spin-qubits in quantum dots, cond- mat 0110150 (2001)

16. Solano E., Santos M.F., and Milman P.: Quantum phase gate with selective interaction, qu-phys 0102052 (2001)

17. Stein D.L.: Spin glasses: Still complex after all these years? Lecture Notes in Physics 633, 349-361 (2003)

18. Thorwat M., and Haenggi P.: Decoherence and dissipation during quantum XOR gate operation, cond-mat 0104513 (2001)

19. Timonin P.N.: Thermodynamics of strongly frustrated magnet in a field: Ising antiferromagnetic on triangular lattice, arXiv:1005.2650v1 (2010)

20. Vidal G., Masanes L., and Cirac J.I.: Storing quantum dynamics in quantum states: syochastic programmable gate for $\mathrm{u}(1)$ operations, qu-phys 0102037 (2001)

21. Zhou X., Leung D., and Chuang I.: Methodology of quantum logic gate construction, qu-phys 0002039 (2000) 